\title{
High Throughput Tender 3D X-ray Imaging of Cells for Correlative Microscopy
}

Katarzyna Matusik, Sylvia Lewis, David Vine, Jeff Gelb and S.H. Lau

Sigray, Inc., Concord, California, United States

X-ray microscopy has emerged in the past few years, driven by intrinsic advantages of nondestructive high resolution imaging and enabled by major advances in high-resolution x-ray optics and sources. The technique has become the leading 3D nano-imaging approach for intact cells in their native or nearnative hydrated state [1] and has facilitated bridging the critical resolution gap between electron and light-based approaches (Fig. 1) [2].

One of the major advantages of the x-ray microscopy is its potential for correlative imaging [3-5]. X-rays provide $3 \mathrm{D}$ information about whole, hydrated cells without requiring staining or serial sectioning, which makes it an ideal correlative technique. This includes as a structural context for interpreting cryo super resolution light microscopy results [5-6] that lack structural context and as a complement to electron microscopy for aligning the hundreds of slices required for a large cell or to prescreen cells or regions of interest [7].

Laboratory and synchrotron-based cellular x-ray microscopes have primarily operated in the "water window" (285-543 eV) [8]. In the "water window," water is relatively transparent because the x-ray energies are less than the oxygen K-shell absorption edge energy of $543 \mathrm{eV}$, but carbon-containing organic matter is highly absorbing because the x-ray energies are above the carbon K-shell absorption edge energy of $284 \mathrm{eV}$. Such water window systems have provided powerful capabilities for imaging prokaryotic (e.g. yeast, bacteria, algae) cells and a sub-set of small mammalian cells. However, the system's utility is limited for many medical applications because of fundamental limitations of water window X-rays: it is easily attenuated and has a shallow depth-of-field (DOF) for high resolution imaging, which impedes practical imaging of adherent cells and cells above $10 \mu \mathrm{m}$ in diameter; most mammalian cells are at least $10-20 \mu \mathrm{m}$ in diameter.

Here we present a recent laboratory development using the phase contrast of tender x-rays (e.g. 2-6 keV) for rapid ultrastructural imaging of large mammalian cells at $30 \mathrm{~nm}$ spatial resolution. This system is based on a key recognition that phase contrast between organic matter and water at $\sim \mathbf{2 . 5} \mathbf{k e V}$ x-rays is comparable to or even higher than the respective absorption contrast at $\mathrm{x}$-ray energies in the waterwindow. This realization has motivated developments at synchrotrons (e.g. Diamond Light Source and BESSY) to develop tender $\mathrm{x}$-rays as an option for their water window $\mathrm{x}$-ray microscopes.

The system enables several key advantages for mammalian cell imaging, including:

- $\quad$ Fast 30-minute tomography imaging with 30nm resolution

- $\quad$ High natural phase image contrast between water and organic matter

- $\quad$ Ability to image large (up to $80 \mu \mathrm{m}$ ) unstained cells and fixed stained cells

We will review recent data acquired from Sigray's $2.7 \mathrm{keV}$ BioLambda x-ray microscope system and discuss developments toward facilitating workflows using 3D x-ray tomographies of cells. 


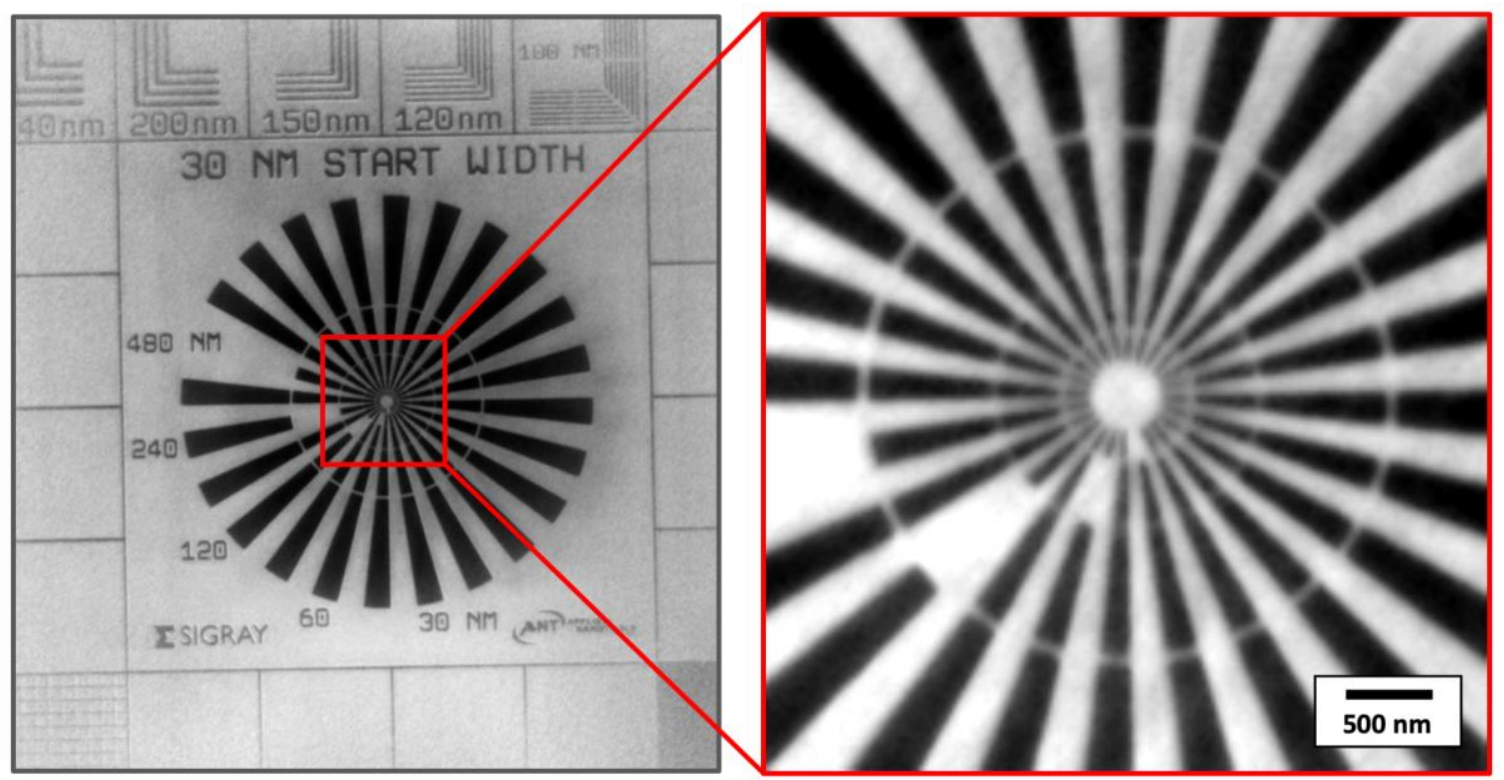

Figure 1. Resolution capabilities of the $2.7 \mathrm{keV}$ x-ray microscope with 30nm spatial resolution

\section{References}

[1] Fogelqvist, Emelie, et al. "Laboratory cryo x-ray microscopy for 3D cell imaging." Nature Scientific Reports 7.1 (2017): 13433.

[2] Yao, Shengkun, et al. "Three-dimensional ultrastructural imaging reveals the nanoscale architecture of mammalian cells." IUCrJ 5.2 (2018): 141-149.

[3] Groen, J., et al. "The cellular landscape by cryo soft X-ray tomography." Biophysical reviews (2019): $1-9$.

[4] Carlson, David B., et al. "Laboratory-based cryogenic soft X-ray tomography with correlative cryolight and electron microscopy." Microscopy and Microanalysis 19.1 (2013): 22-29.

[5] Pereiro, Eva. "Correlative cryo-soft X-ray tomography of cells." Biophysical reviews 11.4 (2019): 529-530.

[6] Fogelqvist, Emelie, et al. "Laboratory cryo x-ray microscopy for 3D cell imaging." Nature Scientific Reports 7.1 (2017): 13433.

[7] Niclis, Jonathan Christos, et al. "Three-dimensional imaging of human stem cells using soft X-ray tomography." Journal of the Royal Society Interface 12.108 (2015): 20150252.

[8] Carzaniga, Raffaella, et al. "Cryo-soft X-ray tomography: a journey into the world of the native-state cell." Protoplasma 251.2 (2014): 449-458. 